\title{
Cognitive Categorization in Hierarchical Systems under Situational Control
}

\author{
Alexander Ya. Fridman \\ Lab. for Systems Modelling, \\ Institute for Informatics and Mathematical Modelling, Kola Science Centre of the Russian Academy of Sciences \\ 24 A Fersman str. \\ Apatity Murmansk region, Russia \\ E-mail: fridman@iimm.ru
}

\begin{abstract}
Methods of cognitive multicriteria classification, generalization of description and coordination of situations in hierarchical subject domains are developed. The methods consider prototypical effects and degrees of typicality of members according to semantic similarity measures of categories modeled in conceptual spaces. This approach also concretizes the notion of situation awareness for the proposed domain model, which allows to increase flexibility of classification and its robustness to data inaccuracy, as well as the speed of search for precedents.
\end{abstract}

Keywords: Cognitive categorization, conceptual space, situational approach, hierarchical system, situation awareness.

\section{Introduction}

In situational control systems proposed by D. A. Pospelov [1], it is required to solve a number of problems based on classification of certain structures or processes. First, it is the task of classifying situations proper where it is necessary to find out to which class the current situation relates, to identify preferences of the decision maker (DM) for further management of the subordinated object and to offer a control impact that implements these preferences taking into account the state of the system and relevant limitations.

Basic information for building a situational control system is grouped as follows:

$Q_{j}, j=1,2, \ldots$ is the current situation at the object;

$U_{k}, k=1,2, \ldots, N_{U}$ is a set of possible single-step impacts;

$S_{i}, \quad i=1,2, \ldots$ is the current complete situation, which includes not only the current situation, but also information about the state of the control system and management strategies, and transfers the object from one current situation to another by implementing a onestep action from the set $U$ :

$$
S_{i} ; Q_{j} \underset{U_{k}}{\longrightarrow} Q_{i}
$$

Secondly, it is the task of generalizing description of situations on the basis of their classification according to a set of pragmatically important features [1]. In all cases when developing recommendations, it is desirable to consider previous management experience, that is, to be able to analyze archives of situations and identify analogs of the current situation there. Due to complexity of the managed system, it makes no sense to rely on availability of complete analogs, which leads to the need of involving intelligent decision-making techniques. In particular, it looks reasonable to advance from analyzing classical categories of structures and processes based on equivalence relations to methods of cognitive (semantic) categorization proposed in [2] and received considerable development $[3,4]$ by taking into account prototypical effects and degree of typicality of category members [5], as well as using semantic similarity measures for categories modeled in so-called conceptual spaces [4]. In the present article, a solution of the above-listed problems is considered within the framework of the previously developed system of situational modeling (SMS) for dynamic hierarchical spatial complexes (DHSC) [6-8]. SMSs can be established at various organizational levels (federal, regional and corporate ones) to manage DHSCs of relevant territorial scales.

Situational approach is used in different subject domains and formalized in various ways $[1,9,10$, etc.]. In general, complete situation is described in three main aspects: knowledge of the current structure of the managed object, the current state of the control system, and the technology (strategies) of control [1]. Thus, each situation is described by a set of values of 
variables characterizing the listed aspects. The basis of "classical" situational control is a semiotic (symbolic) model constructed as a network, at the nodes of which there are internally consistent formal models, and transitions between nodes are specified by rules for changing parameters of formal models called correlation or logical-transformation rules [1].

To our mind, complexity of situational control can be significantly reduced by adapting it to specific models of subject domains. SMS provides a formal definition of the concept "situation" and the main problems of situational approach for a specific DHSC model, as well as a solution for tasks of classifying and generalizing situations. However, it is still difficult to construct classes of situations there because of insufficiently flexible classification procedure.

Currently, the concept of situation(al) awareness (SA) [10-12, etc.] describes the most general principles of information preparation and processing for implementation of situational approach in dynamic subject domains. Situational awareness involves understanding what is happening in the environment, in order to know how information, events and actions will affect goals and objectives at the current moment and in near future. Insufficient or incorrect awareness of the situation is considered one of the main factors associated with accidents that are caused by the "human factor" [10]. Thus, awareness of the situation is especially important in professional activities, where the flow of information can be quite high. However, in our opinion, SA is also essential for considered problems of DHSC modeling, where time for decision making is sufficiently large, but other aspects of SA are very important.

Formal definition of SA is divided into three segments: perception of elements in the environment, understanding of the situation and prognosis of future status [12]. The presence of a complete, accurate and relevant SA is especially important when complexity of the process and the situation provokes apprehension on the DM's ability to deal with this independently.

The fundamentals of SA principles proposed by M. Endsley, the author of this approach (for example, [12]), can be summarized as follows:

1) perception, comprehending and forecasting as three basic levels of SA;

2) significant role of goals and targeted data processing in focusing attention and interpreting significance of perceived information;

3) role of information specificity in "capturing" attention with consideration emerging data (data- driven) as well as importance of alternation between goal-driven and data-driven processing;

4) role of expectations (fueled by current situation model and information from long-term storage) in focusing attention and interpretation of information;

5) strict requirements for limiting working memory in order to narrow SA for beginners and DMs in new situations, but at the same time, enormous advantages of mental models and comparisons of samples with a prototypic scheme that largely bypass these limitations;

6) usage mental models to provide means for integrating various aspects of information and understanding its meaning (in relation to goals), and to support people's ability to compile useful forecasts of likely future events and conditions;

7) matching templates with schemes, i.e., prototypic states of mental models, which provides rapid comprehending and forecasting capabilities related to the recognized situation, and in many cases - extraction of adequate situations by a one-step action.

Some researchers criticize this approach for excessive generality (in particular, see [11]). General principles of SA seem to really become realistic only in relation to a specific model of decision-making in a particular subject domain. Therefore, they are further interpreted for SMS.

In connection with the foregoing, subsequent sections of this paper explore possibilities of using cognitive categorization to develop situational approach to studying dynamics of spatial objects on the basis of their conceptual model, which is the core of SMS [7].

\section{Synopsis of Situational Modeling}

In SMS, objects form a hierarchy that reflects organizational links among DHSC components. During construction of the model, objects are associated with an electronic map (GIS) in such a way as to ensure a one-to-one correspondence between the conceptual and geographical representations of a DHSC. The links between model elements are formalized by resources that simulate material and informational interactions of these elements and represent time series of data. Processes describe nature of resource transformations within model elements. To implement multi-model approach in the course of developing the model, a number of types of processes are allowed, at present they are computational modules of a sufficiently arbitrary structure (generally described by difference equations), user functions or sets of production rules stored and processed in a specialized expert system (ES) incorporated into SMS. The type of a process is 
set by assigning an executor, which determines the computer implementation of the process. Executors are also appointed to resources external for the DHSC under study, their time-related values can be formed by means of user functions, external databases or ES rules. Alternatives to implement the DHSC that need to be compared are introduced into the model by decomposing objects by means of classification relation (OR) and/or specifying several options for obtaining resources from DHSC elements that generate them.

The DHSC conceptual model (hereinafter situational conceptual model - SCM) is built by declaring a hierarchy of objects, specifying resources generated and consumed by these objects, as well as processes simulating resource transformations within objects. At all stages of construction and maintenance of SCM, a detailed formal control of its correctness is conducted [6], which looks to be the only possible means of developing realistic models for complex systems. To improve efficiency of the correctness control, frames of model elements are supplemented with special attributes (types and categories) that are set by a user or assigned automatically during creation or modification of SCM based on analyzing positions of these elements in the model. Such attributes specify requirements to interrelationships of SCM elements and allow to detail the correctness control to the level of an individual resource.

The situational approach to investigating a DHSC is implemented in SMS as follows.

An elementary form of information representation in SMS is a fact that contains data about values of a certain resource. The initial situation is entered by a user in the form of a finite set of facts. The SMS ES analyzes SCM and the initial situation, and redefines the latter up to a complete situation. Every complete situation corresponds to a connected SCM fragment, which may include alternative options for the DHSC structure. A sufficient situation is one of choiceless options to model the corresponding complete situation. Sufficient situations can be compared with each other both statically, according to specified quality criteria in the course of classification of situations, and dynamically in the simulation mode. A scenario is a sequence of sufficient situations generated from the same complete situation; it determines a specific simulation variant.

Thus, SMS integrates various forms of knowledge representation (computational data, graphical characteristics of DHSC elements, expert knowledge) for solving the problem of investigating and forecasting behavior of DHSCs as multilevel multicomponent spatial objects.

SMS is attributed with the following features [6]:

- automation of all stages of modeling;

- wide application of expert knowledge;

- usage of semantically significant notions of a subject domain for constructing its formal description;

- use of GIS-technology not only for graphical representation of DHSC components and simulation results, but also for setting a task for simulation and performing spatially dependent calculations;

- support of modern scenario approaches to modeling;

- availability of detailed formal analysis of the model;

- advanced techniques for comparative analysis of situations in statics and dynamics;

- automatic synthesis of the simulation execution environment;

- usage of common instrumental environment and methodology for modeling both normal and critical modes of DHSC functioning;

- possibility of integrating expert knowledge on various aspects of DHSC functioning to justify decision making.

Thus, SMS provides a DM with algorithmic support to substantiate decisions on changing or maintaining the structure of the subordinated object, and is an alternative to an expert council in this sense.

Now we will consider some issues of processing situations in SMS in some more detail.

\section{Classification and generalization of situations}

In the pilot version of SMS [7], situations were classified in accordance with a numerical generalized performance criterion assigned to any SDHC element. This criterion has the form of a weighted sum of scalar (particular) quality criteria with importance coefficients inversely proportional to tolerances for deviations of particular criteria from their nominal value, which corresponds to common sense: the more important is a particular criterion for a DM, the lesser tolerance is advisable to assign to deviations of this criterion. Advantages of the proposed generalized criterion are as follows. It is equal to unity when values of all its arguments (particular criteria) are on the verge of tolerances and does not exceed unity if all arguments are within their tolerances. For its formation, DM should only specify semantically understandable variables, namely nominal values of particular criteria and tolerances for their deviations. In order to find out 
the source of ineffective functioning of HDSC, it is sufficient to identify the object that is both the furthest from the root of the modeled fragment and has the generalized criterion significantly exceeding unity. In SMS, such a root is called the decisive object (DO) since a DM is seated there according to the essence of the current task. Calculating the values of this criterion up the tree of objects from the leaf objects to DO allows to uniquely classify sufficient situations by placing in one class those situations in which the same particular criterion makes a minimal contribution to the generalized criterion, that is, this particular criterion is satisfied to the maximal degree. Inside one class, it is natural to consider a sufficient situation the better, the less value of the generalized criterion it has.

However, if it is necessary to transfer DHSC into another class of situations, it is required to advise the DM such a sufficient situation from a new class that is minimally different in structure from the current situation, so as not to introduce excessive perturbations into the real system, but still close enough to the best structure of the required class. The generalized criterion presented above is not directly suitable for solving such a problem, since its values depend not only on the structure of the situation, but also on the values of involved resources. Therefore, below we propose a cognitive technique for classifying situations in SMS, which depends less on the values of resources exchanged between the components of HDSC.

\subsection{Cognitive Categorization in SMS}

Works of psychologists (for example, [2]) show that a person constructs categories (classes) of entities of the surrounding world substantially differently from the construction of mathematical classes based on the relation of equivalence. The main difference is that all elements of mathematical classes are equally typical for this class, and "human" (cognitive) categories have an internal structure, that is, they incorporate more and less typical members. Cognitive approach to the problems of artificial intelligence was investigated both abroad (for example, [13]), and in Russia [14]. It turned out to be effective in different tasks, therefore we suggest to apply it for solving the above-introduced problems of classifying situations in SMS in order to reduce dependence of classification results on the specific values of resources under which the classification is carried out as well as to depress perturbations in the real system resulted from changes in its structure.

The idea of cognitive classification of situations in SMS is as follows. In the course of the classification, we identify not only one object structure optimal in a given class of situations (it is, of course, a prototype of this class in the terminology of cognitive categorization), but several structures that differ insignificantly (by 10-15\%) from the optimal one by the mentioned numerical performance criterion. We call such structures preferable in this class. In order to weaken the dependence of classification results on the resource values used during classification, we calculate the semantic distance (for instance, [3]) between preferable structures and archive only those for which this distance is small $(10-15 \%$ of the maximum distance in the class). We perform a similar cognitive classification for all classes obtained by the numerical criterion and create a complete archive of preferable situations, which stores their groups for each possible (for a given DO) class. Then calculate the same distance between all pairs of preferable situations of different classes and select pairs with the minimum semantic distance between them. As a result, the mapping (1) for the problem of situational control of the structure of the modeled system will be constructed. Let us call it cognitive mapping of situations (CMS).

Cognitive classification of situations can be made a priori, before the actual system is in operation, and then refined by experimental data. The decision-making procedure during the operation of the object should include the following steps:

- classification of the current situation by the numerical criterion;

- searching the archive of preferable situations for the situation of the obtained class, which is the closest to the current one by semantic distance;

- selection from CMS the pair of the found preferable situation found in the class that DM has appointed to continue the operation of the object; implementation of the preferred situation for a new class on the object.

To complete this section, we only need to propose a metrics for constructing CMS.

\subsection{Geometric Similarity Metrics for Hierarchies}

To date, there exist a lot of measures of semantic similarity or distance between vertices of hierarchical structures constructed of objects - terms and concepts of a subject domain (see, for example, [3, 4]). They can be divided into geometric ones, where the object is considered as a point in the properties space, and semantic metrics proper developed for hierarchical objects (taxonomies and ontologies). The latter are based on the path length between vertices of the object graph. Such metrics are not relevant to the task at hand, since situational control requires to assess structural 
differences between descriptions of a situation as a whole. Therefore, we propose to modify the Tversky's ratio model (TRM) [15] for hierarchical objects.

In TRM, the distance between two objects $a$ and $b$ is defined as following fraction:

$S(a, b)=f(A \cap B) /[f(A \cap B)+\alpha f(A-B)+\beta f(B-A)]$,

where $A$ and $B$ are some sets of properties of these objects; / denotes the symbol of division of numbers; minus stands for difference of sets; $f$ is a function; nonnegative $\alpha$ and $\beta$ represent weights of common and different properties of objects. In most methods, the power of a set of arguments is used as the function $f$.

Formula (2) is not directly suitable for classifying situations, since it does not take into account the hierarchy of description of a situation postulated in [1]. As it seems natural to assume that the contribution of distinctions in properties at lower levels of a hierarchy should be smaller than of distinctions at higher levels, we will evaluate the structural similarity of a hierarchical object as

$$
\frac{1}{N} \sum_{i=1}^{N} \frac{1}{i} S_{i}
$$

where $i=1,2, \ldots N$ is the level number in the hierarchy, $N$ is the total number of levels, and each $S_{i}$ is calculated by (2) for subsets of properties included in the description of this level. Let us call the metrics (3) hierarchical Tversky's metrics (HTM). When comparing hierarchical structures, the sets $A_{i}$ and $B_{i}$ include names of objects of level $i$. Here the hierarchical description of situations is considered by normalizing TRMs values with weight coefficients inversely proportional to the level number. By introducing some additional weights for summands in (3), it is possible to allow expert opinions on relative importance of the levels.

The relation (3) is a linear convolution of several TRMs; therefore it satisfies all the requirements usually imposed on measure functions.

To explain formula (3), we will analyze a hypothetical complete situation, represented as the AND / OR-tree of SMS objects in Fig. 1, where the arcs label OR-vertices, the root object (DO) has the level 0 . For symmetry, let $\alpha=\beta=1$ in (2). The sets $A_{i}$ and $B_{i}$ include objects $O_{i j}$ of the level $i$.

The complete situation in Fig. 1 contains several alternative sufficient situations [6-8] (four of them are shown in Figures 2-5), which should be classified according to the HTM criterion (3). It is not difficult to calculate measures of pairwise similarity for these situations with respect to powers of sets of common and different objects (the subscripts correspond to numbers of situations):

$$
S_{13}=0.54 ; S_{12}=S_{14}=S_{23}=0 ; S_{24}=0.5 .
$$

The resulting values of similarity measures do not contradict intuitive perception: the situations in Figures 2 and 4 are fairly similar, as are the situations in Figures 3 and 5, while other pairs of situations (for example, in Figures 2 and 3) are not similar at all.

We suppose to use $n$-tuple algebra [16] as a means to classify situations in SMS.

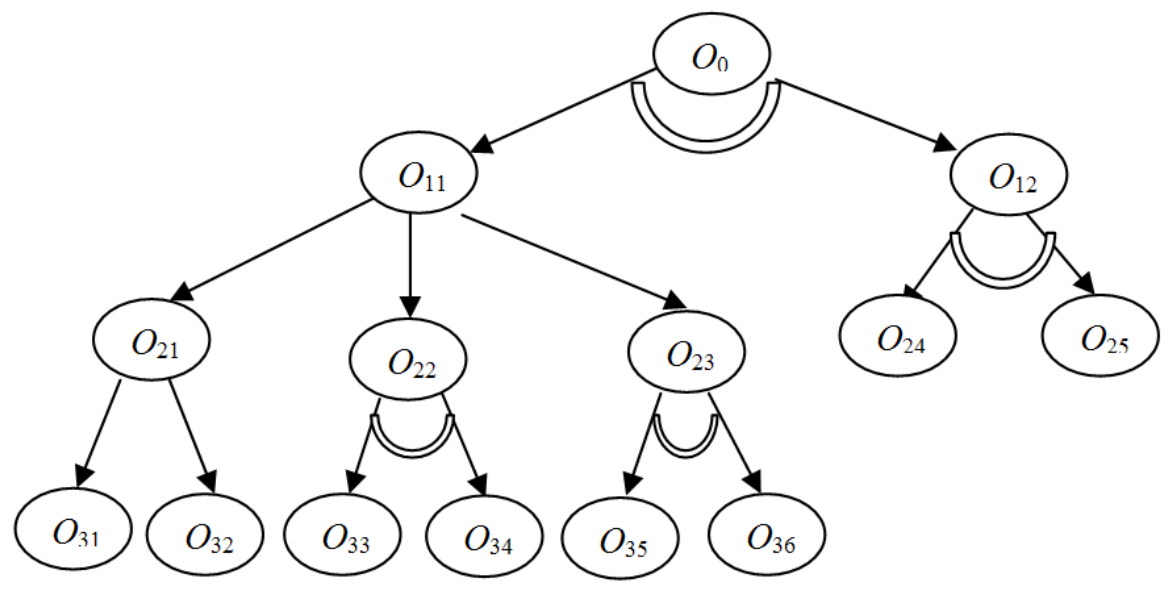

Fig. 1. A complete situation. 


\subsection{Situational Awareness in SMS}

The above-introduced HTM (3) provides a good basis for dealing with situations in SMS. However, additional studies have shown that prior to classification, it is necessary to form and carefully preprocess the archive of situations stored in SMS memory. What follows are principles of layout and selection of situations for storing in the SMS archive.

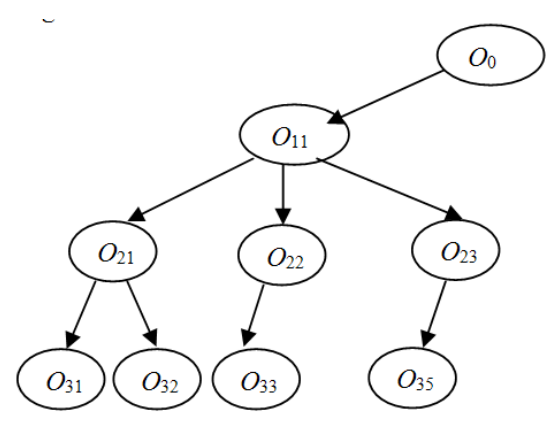

Fig. 2. The sufficient situation 1.

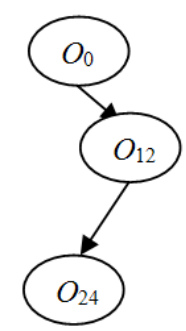

Fig. 3. The sufficient situation 2.

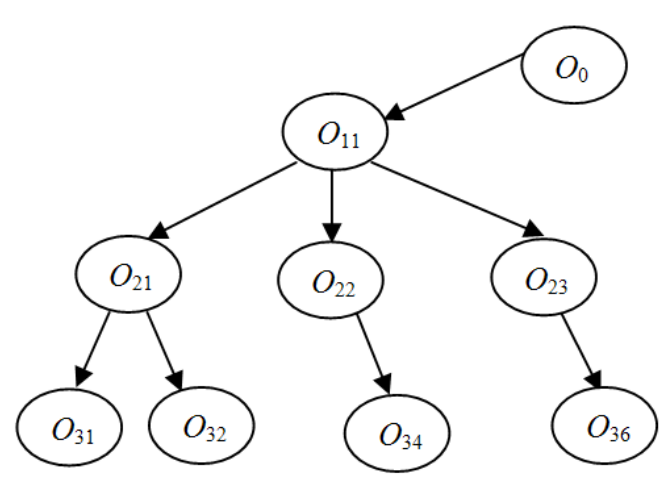

Fig. 4. The sufficient situation 3.

Naturally, this archive should contain all real SMS scenarios in the given application, but the main array of information is created a priori based on results of simulation experiments with SCM. After simulations, the archive should contain sets of preferable situations for all classes specified by criterion (3) for all DOs included in SCM. This goal requires for solving the tasks described below.

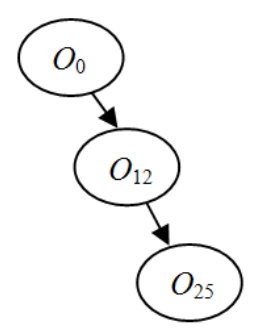

Fig. 5. The sufficient situation 4 .

\subsubsection{Study of Stability of Scenarios}

In order to increase stability of simulation results to uncertainties in initial data, we propose to use an approach analogous to methods of theory of sensitivity, which is used in automatic control (for example, [17]). In order to reduce influence of uncertainties regarding parameters of SCM and thereby improve accuracy of forecasts under small changes of source data, it is advisable to select the scheme and parameters of data preprocessing during simulation according to additional criterion of minimizing the influence of small variations in initial data on the values of the above-mentioned generalized numerical criterion. We call this additional criterion the criterion of controls quality (CCQ). Similar requirements are imposed on projected algorithms and systems in various fields of knowledge: regulators with reduced sensitivity and robust systems in automatic control, "rough" (crude) models in modeling theory, etc. The general scheme of the CCQ application is as follows: after synthesis of an object model (or any of its essential parameters at intermediate stages of modeling) with using the generalized criterion for a set of experimental data, we accomplish a certain modification of this set, re-synthesis and evaluation of changes in the generalized criterion after these changes in data. Based on results of this assessment, a conclusion is made on applicability of the synthesized model. A model is considered acceptable on CCQ if specific value of a change of the generalized criterion is comparable with relative changes in source data caused this change.

\subsubsection{Generalization of Situations}

With the help of HTM it is also possible to carry out generalization of situations in SMS.

Theory of situational control [1] considers basically important to develop procedures for generalizing descriptions of situations on the basis of their 
classification with using a set of pragmatically important properties; this set is subject to synthesis as well. The theory also points out the following fundamental features of concept formation and classification in situational control:

existence of generalization procedures based on the structure of relations between elements of situations;

ability to work with names assigned to individual concepts and situations;

need to harmonize classification of situations on a basis of classification on the set of impacts (controls).

To implement these principles, we further propose a procedure to apply HTM.

As a result of classification of situations by the numerical criterion, a large number of classes of situations are generated for different DOs and various leaf objects of these situations, which unambiguously determines every situation due to the tree-like decomposition of objects in SMS SCM. After culling out situations that do not meet the stability requirement according to CCQ, we use HTM to form classes of preferable situations for each DO. Source data for this are: types of leaf objects and DO for sufficient situations under study, and the result of classification is a unique type of a sufficient situation supplemented by a sequence number of the class to which the current sufficient situation belongs and its number within this class. The sequence number of the class is determined by the number of the resource that dominates in this class by the numerical criterion for this DO, and the sequence number of the situation within the class is given by its preference according to this criterion. The best (prototypical in terms of conceptual spaces) sufficient situation for each class is assigned to the sequence number 1 .

Generalization includes two main steps: search for common features of situations that fall into one class for each studied SCM fragment, and search for inclusions of situations into a situation of higher levels (the level number here is set by the level of DO), which is carried out by means of Graph Matching (for example, [18]). The results of generalization are formed as logicaltransformational rules [1] of the SMS ES [6], which include both positive and negative examples. Such rules control the entire process of classification of situations.

Then, effectiveness of alternatives presented in SCM is estimated. It is the higher, the wider set of classes of situations includes different variants of an alternative. Then alternatives are ordered according to this criterion, and DMs are first of all presented with the most effective alternatives for evaluation. The opposite statement is also true: an alternative is ineffective for a given DO, if none of the available options of choice in this alternative transfers sufficient situations into another class. Results of effectiveness evaluation of alternatives are also formalized as rules of the SMS ES.

\subsubsection{Features of SA in Situational Modeling}

Let us give a concretization (under the same ordinal numbers) of the SA principles listed in Introduction:

1) perception is modeled by setting an initial situation; comprehending is provided by forming a complete situation and determining the organizational level to solve the current problem within SCM; forecasting is implemented by simulation experiments;

2) a DM sets the goal of modeling by selecting the desired class of situations during simulation;

3) specificity of information is taken into account by choosing an initial situation and automatic rejection of unpromising alternatives;

4) expectations of a DM in SMS are formalized by selecting a dominant particular criterion in the generalized criterion and a set of permissible alternatives stipulated in SCM when it is created.

The remaining principles of SA are related to mental model of a subject domain, which is defined in SMS by means of SCM. In this case, an important role is played by principles of cognitive categorization, described in Section 3.1.

\section{Coordination and Planning of Interactions for DHSC Components}

As already noted, the problem of coordination arises when there are several DMs responsible for functioning of different components of one DHSC. To solve it, it is required to find conditions under which all DMs reach their local goals and simultaneously solve the top-level task for which the whole system was created. Traditionally, this problem is considered for a two-level system, since the possibility of generalization to more levels is obvious [19]. Coordination has two aspects: coordination of the tasks of DMs from the lower level with respect to the task of the Coordinator (the top level DM) and coordination with regard to the global goal of DHSC. In SMS, on the basis of the interaction prediction principle [19], necessary coordinability conditions for both these aspects were found by analyzing gradients (increments for a discrete description of the problem) of the mentioned in previous sections generalized numerical quality criterion based on the nominal values of particular criteria [6]. In [20], the same approach was applied to coordination of teams of intelligent dynamic systems (IDS) [21]. IDSs are 
fundamentally different from "classical" dynamic systems since they are implemented using databases and knowledge bases and allow for presence of nonnumerical components in their state vector. Nevertheless, principle of predicting interactions turned out to be suitable too, but instead of gradients of the criterion, it is necessary to analyze inclusion for state vectors of the IDSs that are members of a team.

Using the same approach and the notion of $\mathrm{N}$-attainability [21], it occurred to be possible to solve the planning problem in SMS and in collectives of IDSs, that is, to propose algorithms for selecting controls that transfer the system from an initial state to a target state [20]. Planning is a necessary stage preceding any management.

\section{Conclusion}

In the process of developing SMS, a wide range of issues related to effectiveness of modeling and decision making within this system was considered and solved, namely classification and generalization of situations, coordination and planning, etc. This witnesses prospective contribution of situational approach into the field of creation modern intellectualized control systems for complex dynamic objects.

\section{Acknowledgements}

This work was supported in part by the Russian Foundation for Basic Researches (grants 16-29-04424, 16-29-12901, 18-07-00132, 18-01-00076 and 18-2903022).

\section{References}

1. D. A. Pospelov, Situational Control: Theory and Practice (Columbus, OH: Batelle Memorial Institute, 1986).

2. E. H. Rosch, Natural categories. Cognitive Psychology. 4(3) (1973) 328-350.

3. P. Gärdenfors, The Geometry of Meaning: Semantics Based on Conceptual Spaces (Cambridge, MA: MIT Press, 2014).

4. F. Zenker and P. Gärdenfors P. (eds.), Applications of Conceptual Spaces. The Case for Geometric Knowledge Representation (Synthese Library. Springer, vol. 359, 2015).

5. L. Decock and I. Douven, What is Graded Membership? Noûs. 48 (2014) 653-682.

6. A. Ya. Fridman, Situational structure control of industrynatural systems (Saarbrucken, Germany: LAP, 2015). (In Russ.).

7. B. Sokolov and A. Fridman, Integrated Modelling Environment for Decision Making Support in Supply Chain Management: Conceptual Approach, in Proc. of the $13^{\text {th }}$ IFAC Symposium on Information Control
Problems in Manufacturing (Moscow, Russia, June 3-5, 2009), pp. 598-603.

8. I. L. Artemieva and A. Ya. Fridman, Ontologies in the Automation Problem for Situational Modelling, Information Technology in Industry, 2017 (in press).

9. W. Wimmer et al., ECODESIGN - The Competitive Advantage, Alliance for Global Sustainability (Bookseries 18, Springer Science+Business Media B.V., 2010).

10. J. Lundberg, Situation Awareness Systems, States and Processes: A holistic framework, Theoretical Issues in Ergonomics Science. 2015.

11. S. Banbury and S. Tremblay, A cognitive approach to situation awareness: Theory and application (Aldershot, UK: Ashgate Publishing, 2004), pp. 317-341.

12. M. R. Endsley, Final Reflections: Situation Awareness Models and Measures, J. of Cognitive Engineering and Decision Making. 9 (1) (2015) 101-111.

13. P. Gardenfors and S. Lohndorf, What is a domain? Dimensional structures versus meronomic relations. Cognitive Linguistics 24 (3) (2013) 437-456.

14. O.P. Kuznecov, Cognitive semantics and artificial intelligence, Artificial Intelligence and Decision Making. 4 (2012) 32-42. (In Russ.).

15. A. Tversky, Features of similarity, Psychological Review 84 (1977) 327-352.

16. B. Kulik and A. Fridman, N-ary Relations for Logical Analysis of Data and Knowledge (IGI Global, 2017).

17. M. Eslami, Theory of Sensitivity in Dynamic Systems, An Introduction (Heidelberg, Germany: Springer-Verlag, 1994).

18. K. Sambhoos, R. Nagi, M. Sudit, and A. Stotz, Enhancements to High Level Data Fusion using Graph Matching and State Space Search, Information Fusion 11 (4) (2010) 351-364.

19. M. D. Mesarovic, D. Macko, and Y. Takahara, Theory of Hierarchical Multilevel Systems (New-York and London: Acad. Press, 1970).

20. A. Fridman, Planning and Coordination in Hierarchies of Intelligent Dynamic Systems, TELKOMNIKA 14 (4) (December 2016) 1408-1416.

21. G. Osipov, Intelligent dynamic systems, Scientific and Technical Information Processing 37 (5) (December 2010) 259-264. 